\title{
PENSATA
}

\section{DECISÕES CORPORATIVAS EM GRUPO: UMA ABORDAGEM COMPORTAMENTAL}

É comum que decisões organizacionais importantes sejam tomadas em grupo. Nas instituições financeiras, não raro, as concessões de crédito são decididas em comitês. Na esfera governamental, decisões fundamentais, tais como a definição da taxa de juros básica da economia, também são tomadas em colegiado. Nas grandes empresas privadas, o conselho de administração (CA) está no topo da hierarquia organizacional e tem a palavra final sobre estratégias de investimento, financiamento e fusões e aquisições. Todavia, as peculiaridades do processo decisório grupal são largamente ignoradas na literatura de finanças, efetivamente tratando-se o coletivo como se fosse um indivíduo. Por exemplo, as pesquisas no campo de finanças corporativas comportamentais enfatizam os processos cognitivos e vieses do decisor individual e dão pouca atenção a como esses processos interagem para produzir a decisão do grupo (para uma revisão dessa literatura, vide Baker \& Wurgler, 2013). Neste ensaio, apresento, de maneira concisa e seletiva, o estado atual da discussão multidisciplinar emergente sobre o processo decisório em pequenos grupos, com ênfase em seus aspectos comportamentais. Primeiramente, abordo as vantagens e dificuldades da decisão em grupo em comparação com a decisão individual. Em seguida, apresento contribuições recentes que mostram como a qualidade da decisão do grupo depende do contexto e como pequenas alterações do ambiente decisório podem ter consequências relevantes.

\section{O GRUPO E 0 INDIVÍDUO}

Em recente revisão da literatura de psicologia experimental e áreas correlatas, Tindale e Kluwe (2015) mostram que decidir em grupo traz resultados melhores do que decidir individualmente em muitos contextos. Grupos encontram com mais facilidade soluções corretas para problemas; produzem previsões mais precisas e ideias mais criativas; obtêm melhores resultados em negociações e em exames acadêmicos; tomam decisões de contratação superiores; e recuperam informações de maneira mais precisa (i.e., possuem uma "memória coletiva" superior à memória individual).

Uma das linhas de pesquisa mais antigas e consolidadas nessa literatura investiga o fenômeno da "sabedoria das multidões", concluindo que grupos são mecanismos eficientes de agregação de opiniões e estimativas individuais mesmo quando seus membros não interagem uns com os outros. Por exemplo, Ariely et al. (2000) demonstram que, sob certas condições estatísticas, a média de estimativas de probabilidade produzida por um grupo será sempre superior à estimativa individual de qualquer dos seus membros, argumento corroborado por muitos estudos empíricos realizados em condições mais realistas.

É provável que a natureza social da decisão seja uma resposta adaptativa às condições que moldaram a evolução da vida em sociedade. 0 processo decisório coletivo pode ter contribuído para mitigar limitações cognitivas individuais e, dessa forma, aumentar os recursos disponíveis para todos. Nessa linha, Hastie e Kameda (2005) simulam a escolha de locais apropriados para caça por grupos Faculdade de Economia,

Administração e Contabilidade, São Paulo, SP, Brasil 
de caçadores-coletores. Comparando diversas regras de decisão alternativas, os autores relatam que regras simples de maioria/ pluralidade (e.g., escolher o local de caça com maior número de votos entre os membros do grupo) têm desempenho ótimo e despendem poucos recursos cognitivos, inclusive superando de modo consistente a performance de regras baseadas no melhor decisor individual. Algumas pesquisas sugerem que processos similares podem beneficiar também outras espécies. Por exemplo, Kameda, Wisdom, Toyokawa e Inukai (2012) reportam que abelhas aparentemente escolhem locais para a construção de colmeias com base em regras de maioria/pluralidade.

Infelizmente, entretanto, a boa performance das decisões grupais na vida real não é garantida. Há consistente documentação de anomalias e disfunções em decisões coletivas, tanto em contextos experimentais quanto em estudos de caso de decisões reais de grande importância (e.g., a decisão de comitê que antecedeu a explosão do ônibus espacial Challenger). Por exemplo, em diversos contextos experimentais, grupos tendem a tomar decisões mais questionáveis eticamente em comparação com indivíduos (Nikolova, Lamberton, \& Coleman, 2017). Outros experimentos documentam um efeito de polarização que leva grupos a tomarem decisões mais extremadas (mais conservadoras ou mais arriscadas) do que tomariam seus membros individualmente (Bainbridge, 2002).

As causas das falhas em decisões colegiadas são variadas e controversas na literatura acadêmica. Tindale, Smith, DykemaEngblade e Kluwe (2012) argumentam que os mesmos processos decisórios grupais podem conduzir a decisões superiores ou inferiores àquelas tomadas individualmente. Os autores enfocam especificamente os efeitos de dois tipos de compartilhamento social: compartilhamento de preferências e compartilhamento de representações de tarefas (task representations). 0 primeiro remete ao grau de alinhamento dos membros do grupo com relação a preferências por alternativas de decisão e o segundo remete ao grau de compartilhamento de quaisquer conceitos, normas, perspectivas ou processos cognitivos relevantes para uma determinada tarefa ou situação (e.g., procedimentos utilizados para encontrar a alternativa "ótima" entre as consideradas). Tindale et al. (2012) propõem e verificam experimentalmente que as decisões do grupo podem ser piores do que a decisão média individual quando as representações de tarefas compartilhadas por seus membros são enviesadas, por exemplo, quando o modelo compartilhado utilizado para processar as informações e ranquear alternativas é inapropriado para o problema em questão. Em contextos desse tipo, discussões em grupo tendem a exacerbar ao invés de mitigar os vieses e limites cognitivos individuais. Por exemplo, Tindale e Kluwe (2015) revisam estudos nos quais as distorções das decisões associadas a alguns dos vieses individuais discutidos em Kahneman, Slovic e Tversky (1982) revelaram-se maiores em grupos do que em indivíduos.

Disfunções em processos decisórios grupais também têm sido associadas à conformidade (excessiva) com o grupo. Raafat, Chater e Frith (2009) distinguem duas formas de transmissão de pensamentos entre membros de um grupo: contágio automático e deliberação racional. Um exemplo da última abordagem é a teoria das cascatas informacionais (informational cascades), na qual agentes com informação imperfeita podem racionalmente ignorar suas próprias informações (Bikhchandani, Hirshleifer, \& Welch, 1992). Como ilustração, suponha que, deliberando sobre um relevante investimento estratégico sobre o qual há considerável incerteza, os três primeiros membros do CA a se manifestarem, um depois do outro, defendam a aprovação do projeto. Nesse caso, a teoria prevê que o quarto conselheiro atualizará seu veredito na direção da aprovação do projeto, ignorando parcialmente suas próprias informações. Embora, nesse modelo, a atualização da opinião seja racional, utilizando a regra de Bayes para incorporar a opinião dos colegas que se manifestaram primeiro, a cascata informacional pode causar ineficiência em razão do desconto de informações potencialmente relevantes para a decisão coletiva por parte dos membros do grupo influenciados pela opinião revelada dos colegas.

As abordagens de contágio automático olham para o mesmo fenômeno pelo ângulo de processos não conscientes. Por exemplo, Aydogan, Jobst, D’Ardenne, Müller e Kocher (2017) induzem conformidade em membros de grupos por meio da inalação de oxitocina pelos sujeitos experimentais com o intuito de estudar o comportamento ético em diferentes ambientes. Os autores mostram que a inalação de oxitocina, hormônio associado a comportamentos pró-sociais, aumenta a conformidade intragrupo, e um aumento da conformidade causa redução da honestidade do grupo em ambientes competitivos. Essa evidência alinha-se com os resultados reportados por Nikolova et al. (2017): grupos tendem a se comportar de maneira mais desonesta quando a necessidade de vínculo entre seus membros é maior.

Uma manifestação deletéria da excessiva conformidade ao grupo é o fenômeno conhecido como groupthink, proposto por Janis (1972) e associado a decisões desastrosas tomadas por conselhos e comitês (e.g., a tentativa de invasão de Cuba apoiada pelo governo norte-americano na década de 1960). Entre as distorções do processo decisório associadas ao groupthink, incluem-se a coleta ou compartilhamento insuficiente de informações, falhas de autocrítica e estimações enviesadas de riscos, custos, benefícios e implicações éticas das 
decisões. Embora sua formulação original seja questionada em termos teóricos e as evidências empíricas do fenômeno sejam controversas, o groupthink permanece como uma das ideias mais influentes no debate acadêmico e prático acerca das dificuldades da decisão colegiada (Baron, 2005).

\section{ARQUITETURA DA DECISÃO E DESENVIESAMENTO}

Uma lição da discussão precedente é que a qualidade das decisões em grupo, seja em termos absolutos ou em comparação com decisões individuais, depende inescapavelmente do contexto no qual elas são tomadas. Uma recente literatura multidisciplinar no campo comportamental endereça essa questão, enfocando elementos particulares do que se designa genericamente "arquitetura da decisão".

O estado atual de parte dessa literatura é apresentado por Soll, Milkman e Payne (2015). Em particular, esses autores debruçam-se sobre diferentes procedimentos de "desenviesamento" aplicáveis ao processo decisório individual e/ou grupal. Soll et al. (2015) organizam as diversas contribuições em duas abordagens. A primeira propõe mecanismos que visam a modificar o decisor, por exemplo, por meio de treinamento (e.g., educação financeira), e a segunda enfoca a modificação do ambiente decisório. A segunda abordagem é mais importante para a discussão aqui apresentada, relacionando-se estreitamente com o conceito de nudging, entendido como uma intervenção no contexto da decisão utilizando princípios de psicologia para influenciar o comportamento na forma desejada, porém sem restringir escolhas ou alterar incentivos de maneira significativa (Thaler \& Sunstein, 2008).

A evidência disponível sugere que um mediador fundamental da qualidade do processo decisório de grupos é o grau de compartilhamento de informações. Nessa linha, Tindale e Kluwe (2015) apresentam diversas sugestões de mecanismos aplicados com o intuito de aumentar a probabilidade de que todas as informações relevantes sejam compartilhadas durante as discussões do grupo, incluindo a instituição de regras e procedimentos formais relativos ao compartilhamento de informações, possivelmente envolvendo o estímulo do líder do grupo (e.g., o presidente do CA) à troca de informações entre os participantes da reunião. Também pode ser importante instruir os membros do grupo a não compartilhar suas impressões iniciais ou preferências logo no início das discussões, evitando cascatas informacionais. A mensagem principal dessa linha de investigação é que o foco deve ser direcionado para a informação, e não para as preferências dos membros do grupo, pelo menos no início da reunião, e a ênfase deve recair na acurácia e correção da decisão, e não na busca pelo consenso. Também pode ajudar, nesse ponto, a distribuição da carga informacional por meio da delegação de responsabilidades por tipos específicos de informação a distintos membros do grupo.

Outros mecanismos que contribuem para melhorar a qualidade da decisão, reduzindo o escopo para distorções como o groupthink, envolvem a ampliação do arcabouço decisório para considerar mais alternativas e evidências contrárias (Larrick, 2009). Uma estratégia do tipo é denominada premortem, procedimento que se inicia com a suposição de que determinada proposta em pauta foi aprovada pelo grupo, mas sua implementação foi malsucedida. Em seguida, cada membro contribui para descrever as causas precisas do fracasso hipotético. Finalmente, os insights resultantes do exercício são utilizados para aperfeiçoar a proposta e realimentar a discussão. 0 premortem inclui-se numa classe de estratégias com crescente aceitação prática e acadêmica conhecida como "prospecção retrospectiva" (prospective hindsight) (Soll et al., 2015). Na mesma linha, pode-se programar interrupções em discussões para ajudar a elicitar mais reflexão sobre o objeto da decisão e reduzir a probabilidade de julgamentos precipitados, estratégia especialmente útil na tomada de decisões importantes e infrequentes.

Também é possível mitigar os efeitos deletérios dos vieses de grupo encorajando a integração da opinião de todos os membros, e talvez de pessoas externas, para aproveitar a "sabedoria das multidões", sobretudo quando a decisão envolve estimativas quantitativas (e.g., ranquear alternativas de investimento estratégico por ordem de prioridade). Um refinamento dessa ideia envolve a agregação (e.g., média ou mediana) de estimativas produzidas pelos mesmos indivíduos em diferentes momentos com o intuito de mitigar enviesamentos associados ao contexto temporal da decisão. Tais enviesamentos são mais prováveis quando as estimativas são produzidas em condições de baixa "prontidão decisória" (decision readiness), por exemplo, sob influência de estados emocionais intensos, fadiga ou pressão de tempo (Soll et al., 2015).

Ordóñez, Benson e Pittarello (2015) revisam a literatura sobre os efeitos da pressão de tempo sobre processos decisórios e argumentam que alguma pressão de tempo pode ter efeitos benéficos, ajudando a evitar procrastinação e paralisia por excesso de opções, contribuindo para aumentar a produtividade do decisor. Por outro lado, a evidência científica associa claramente a pressão de tempo severa à redução de criatividade; aumento da saliência de informações negativas; redução de 
processamento analítico; consideração de menor número de opções/atributos; redução de processos cognitivos relacionados a consequências; e aumento de processos decisórios afetivos automáticos, fenômenos que podem conduzir ao aumento da conformidade intragrupo e ao groupthink. Nessa linha, há evidência de que a pressão de tempo se associa positivamente ao uso de estereótipos em contextos de negociação e ao comportamento antiético de grupos, especialmente quando o referido comportamento é facilmente justificável (Ordóñez et al., 2015). Portanto, se a decisão requer empatia com pessoas externas ao grupo ou possui implicações éticas importantes, pode ser crucial desenhar o processo de modo a evitar que ela seja tomada sob pressão de tempo severa.

Finalmente, o mais propalado antídoto contra vieses sociais e má performance de grupos em geral é o aumento da diversidade, isto é, a inclusão no grupo de pessoas capazes de contribuir para o processo decisório a partir de pontos de vista diferentes associados, por exemplo, a diferenças de formação e conhecimento prévio, gênero ou etnia. Argumentos teóricos e a evidência experimental nessa área sugerem que grupos mais diversos produzem decisões mais acuradas (Davis Stober, Budescu, Dana, \& Broomell, 2014; Tindale \& Kluwe, 2015). Também na literatura organizacional e de governança corporativa, o aumento da diversidade tem sido recomendado como um mecanismo capaz de produzir melhores decisões em ambientes de alta complexidade. Por exemplo, Torchia, Calabrò e Morner (2015) mostram que a "diversidade profunda" (i.e., diferenças de background e de personalidade) se associa a maior criatividade e "conflito cognitivo" (i.e., contribuições a partir de perspectivas e modelos mentais variados) em CAs de empresas norueguesas. Similarmente, os resultados do experimento reportado por Mok e Morris (2010) sugerem que indivíduos com identidades culturais conflitantes (e.g., subgrupos de imigrantes ou de indivíduos com vivências relevantes em culturas distintas) resistem mais a consensos quando estes são incorretos, reduzindo o risco de groupthink.

A evidência disponivel sugere, em particular, que vieses de conformidade podem ser mitigados pela diversidade de gênero. Por exemplo, Nikolova e Lamberton (2016) mostram, em diferentes condições experimentais, que duplas de homens tendem a tomar decisões mais extremadas do que indivíduos e do que duplas de mulheres ou duplas mistas. A diversidade de gênero, nesse contexto, deve reduzir o excesso de conformidade a normas masculinas de priorização de extremos, as quais podem ajudar a explicar a evidência de que grupos frequentemente tomam decisões mais extremadas do que indivíduos (Bainbridge, 2002).
Por outro lado, assim como as demais soluções para os problemas da decisão em grupo, aumentar a diversidade não é panaceia, e sua eficácia dependerá do contexto e da forma como é implementada. Por exemplo, Harvey, Currall e Hammer (2017) descrevem, num minucioso estudo qualitativo, a dinâmica interna de um CA cujas reuniões foram acompanhadas in loco pelos pesquisadores durante cinco anos. Os autores documentam que uma mudança de composição do grupo com o intuito de aumentar sua diversidade de perspectivas e de interesses aparentemente originou um processo disfuncional denominado "desvio decisório" (decision diversion). Nessa dinâmica negativa, os objetivos relacionados ao desempenho eficaz do grupo foram substituídos pela negociação de interesses dos subgrupos que o compunham, resultando na deterioração da qualidade das decisões. Disfunções como essa podem responder pela evidência ambígua acerca da performance de grupos heterogêneos. Logo, aumentar a diversidade pode beneficiar ou prejudicar o desempenho do grupo, dependendo do delicado equilíbrio entre forças que criam divergência criativa e forças que criam conflitos disfuncionais. Nessa linha, Homan, Knippenberg, Kleef e Dreu (2007) mostram, em diferentes condições experimentais, que grupos heterogêneos apresentam melhor desempenho quando seus componentes são treinados e convencidos sobre o valor positivo da diversidade para a decisão grupal.

\section{COMENTÁRIOS FINAIS}

Este ensaio fundamenta-se numa literatura multidisciplinar que investiga padrões de interação sistemáticos em pequenos grupos resultantes de processos cognitivos racionais e não racionais. A evidência científica mostra que esses padrões de interação influenciam a qualidade das decisões do grupo e são influenciados pelo ambiente decisório.

A discussão precedente sugere que intervenções sobre a "arquitetura da decisão" podem contribuir para prevenir disfunções e otimizar processos decisórios colegiados. Entre elas, estratégias que induzem maior compartilhamento de informações e aproveitamento da contribuição de cada membro do grupo; foco na acurácia da decisão; produção de maior número de alternativas; e consideração de evidências contrárias. Entretanto, o que funciona em um caso pode ser contraproducente em outro, como sugere o debate sobre os prós e contras da diversidade intragrupo. Pesquisas futuras deverão contribuir para refinar o conhecimento sobre os condicionantes da eficácia de diferentes estratégias, além de explorar novas possibilidades. Por exemplo, pouco se sabe a respeito dos efeitos potenciais das novas 
tecnologias de comunicação sobre a qualidade da decisão colegiada (para uma discussão especulativa sobre os possíveis impactos da tecnologia, vide Tindale \& Kluwe, 2015).

$\mathrm{Na}$ literatura de governança e finanças corporativas, existe espaço para investigar como as dinâmicas de grupo influenciam as decisões estratégicas e o desempenho das empresas, seja numa perspectiva descritiva ou prescritiva. Parece importante ir além da consideração superficial das estruturas decisórias (e.g., composição básica do CA ou características do CEO) e, de fato, abrir a "caixa-preta" dos órgãos colegiados de alta gestão.

\section{REFERÊNCIAS}

Ariely, D., Au, W. T., Bender, R. H., Budescu, D. V., Dietz, C. B., Gu, H., .. Zauberman, G. (2000). The effects of averaging subjective probability estimates between and within judges. Journal of Experimental Psychology: Applied, 6(2), 130-147. doi:10.1037//1076-898X.6.2.130

Aydogan, G., Jobst, A., D’Ardenne, K., Müller, N., \& Kocher, M. G. (2017). The detrimental effects of oxytocin-induced conformity on dishonesty in competition. Psychological Science, 28(6), 751-759. doi:10.1177/0956797617695100

Bainbridge, S. M. (2002). Why a board? Group decisionmaking in corporate governance. Vanderbilt Law Review, 55(1), 1-55.

Baker, M., \& Wurgler, J. (2013). Behavioral corporate finance: An updated survey. In G. M. Constantinides, M. Harris, \& R. M. Stulz (Eds.), Handbook of the economics of finance (Vol. 2, pp. 357-424). New York, USA: Elsevier.

Baron, R. S. (2005). So right it's wrong: Groupthink and the ubiquitous nature of polarized group decision making. In M. Zanna (Ed.), Advances in experimental social psychology (Vol. 37, pp. 219-253). Cambridge, USA: Academic Press.

Bikhchandani, S., Hirshleifer, D., \& Welch, I. (1992). A theory of fads, fashion, custom, and cultural change as informational cascades. Journal of Political Economy, 100(5), 992-1026. doi:10.1086/261849

Davis-Stober, C. P., Budescu, D. V., Dana, J., \& Broomell, S. B. (2014). When is a crowd wise? Decision, 1(2), 79-101. doi:10.1037/ deco000004

Harvey, S., Currall, S. C., \& Hammer, T. H. (2017). Decision diversion in diverse teams: Findings from inside a corporate boardroom. Academy of Management Discoveries, 3(4), 358-381. doi:10.5465/ amd.2015.0129

Hastie, R., \& Kameda, T. (2005). The robust beauty of majority rules in group decisions. Psychological Review, 112(2), 494-508. doi:10.1037/0033-295X.112.2.494

Homan, A. C., Knippenberg, D. Van, Kleef, G. A. Van, \& Dreu, C. K. De. (2007). Bridging faultlines by valuing diversity: Diversity beliefs, information elaboration, and performance in diverse work groups. Journal of Applied Psychology, 92(5), 1189-1199. doi:10.1037/0021-9010.92.5.1189
Janis, I. L. (1972). Victims of groupthink. Boston, USA: Houghton Mifflin.

Kahneman, D., Slovic, P. \& Tversky, A. (1982). Judgment under uncertainty: Heuristics and biases. New York, USA: Cambridge University Press.

Kameda, T., Wisdom, T., Toyokawa, W., \& Inukai, K. (2012). Is consensusseeking unique to humans: A selective review of animal group decision making and its implications for (human) social psychology. Group Processes and Intergroup Relations, 15(5), 673-689. doi:10.1177/1368430212451863

Larrick, R. P. (2009). Broaden the decision frame to make effective decisions. In E. A. Locke (Ed.), Handbook of principles of organizational behavior (2nd ed.). Chichester, UK: John Wiley \& Sons.

Mok, A., \& Morris, M. W. (2010). An upside to bicultural identity conflict: Resisting groupthink in cultural ingroups. Journal of Experimental Social Psychology, 46(6), 1114-1117. doi:10.1016/j.jesp.2010.05.020

Nikolova, H., \& Lamberton, C. (2016). Men and the middle: Gender differences in dyadic compromise effects. Journal of Consumer Research, 43(3), 355-371. doi:10.1093/jcr/ucw035

Nikolova, H., Lamberton, C., \& Coleman, N. V. (2017). Stranger danger: When and why consumer dyads behave less ethically than individuals. Journal of Consumer Research, 45(1), 90-108 doi:10.1093/jcr/ucx108

Ordóñez, L. D., Benson, L., III, \& Pittarello, A. (2015). Time-pressure perception and decision making. In G. Keren \& G. Wu (Eds.), The Wiley Blackwell handbook of judgment and decision making (pp. 517542). Chichester, UK: Wiley-Blackwell.

Raafat, R. M., Chater, N., \& Frith, C. (2009). Herding in humans. Trends in Cognitive Sciences, 13(10), 420-428. doi:10.1016/j.tics.2009.08.002

Soll, J. B., Milkman, K. L., \& Payne, J. W. (2015). A user's guide to debiasing. In G. Keren \& G. Wu (Eds.), The Wiley Blackwell handbook of judgment and decision making (pp. 924-950). Chichester, UK: Wiley-Blackwell.

Thaler, R. H., \& Sunstein, C. R. (2008). Nudge: Improving decisions about health, wealth, and happiness. New Haven, USA: Yale University Press.

Tindale, R. S., \& Kluwe, K. (2015). Decision making in groups and organizations. In G. Keren \& G. Wu (Eds.), The Wiley Blackwell handbook of judgment and decision making (pp. 849-874). Chichester, UK: Wiley-Blackwell.

Tindale, R. S., Smith, C. M., Dykema-Engblade, A., \& Kluwe, K. (2012). Good and bad group performance: Same process - different outcome. Group Processes \& Intergroup Relations, 15(5), 603-618. doi:10.1177/1368430212454928

Torchia, M., Calabrò, A., \& Morner, M. (2015). Board of directors' diversity, creativity, and cognitive conflict: The role of board members' interaction. International Studies of Management \& Organization, 45(1), 6-24. doi:10.1080/00208825.2015.1005992 